\title{
Complementary pathways in mammalian female sex determination
}

\author{
Serge Nef and Jean-Dominique Vassalli
}

Address: Department of Genetic Medicine and Development, University of Geneva Medical School, 1211 Geneva 4, Switzerland.

Correspondence: Serge Nef. Email: serge.nef@unige.ch

\begin{abstract}
In mammals, the sex of the embryo is determined by the fate of the gonad. Recent papers, including one in BMC Developmental Biology, shed light on the molecular regulation of ovarian development and suggest that the R-spondin $1 /$ Wnt $4 / \beta$-catenin pathway and the Foxl2 transcription factor act in a complementary manner to promote ovarian fate and to repress testicular development.
\end{abstract}

\section{A balance between antagonistic signals in the choice between the male or female pathway \\ Mammalian embryos, like those of most animals, are initially sexually undifferentiated and can develop into either male or female individuals following one of two alternative processes. The paternal transmission of a $\mathrm{Y}$ chromosome triggers testicular differentiation, whereas the presence of a paternal X chromosome pushes gonads towards ovarian differentiation.}

Characterization of the pathways that promote testicular or ovarian differentiation is essential for a better understanding of sex determination pathologies, including gonadal dysgenesis, gonadal agenesis and sex reversal [1]. In humans, $\mathrm{XY}$ sex reversal is relatively frequent (occurring in about 1 in 3,000 newborns) and is genetically heterogeneous, with loss of function of the $S R Y$ male-sexdetermining gene accounting for only $15 \%$ of the cases. By contrast, XX sex reversal is rare (about 1 in 20,000 newborns) and is usually caused by the translocation of $S R Y$ onto another chromosome. The underlying cause of sex reversal still cannot be identified in approximately $75 \%$ of patients, indicating that a significant number of genes (or regulatory regions of known genes) involved in sex determination are yet to be revealed. Several recent reports $[2,3]$, including work by Garcia-Ortiz et al. in $B M C$ Developmental Biology [4], are shedding new light on the genes involved in female sex determination.

The mammalian gonads are derived from the intermediate mesoderm and arise as paired thickenings of the coelomic epithelium on the ventromedial surface of the mesonephros. In mice, the genital ridges are visible at embryonic day 10 (E10) and are composed of somatic cells and primordial germ cells (PGCs). At this stage, the gonad is bipotential - somatic progenitors have the capacity to give rise either to follicular and thecal cells in the ovary or Sertoli and Leydig cells in the testis, whereas PGCs can differentiate either as oogonia or spermatogonia. Sex determination is initiated in the supporting somatic cell lineage by the sex-specific expression of key factors and the subsequent upregulation of downstream male or female pathways responsible for promoting one fate and suppressing the other (Figure 1).

Male sex determination begins with the commitment of Sertoli cells, via the cell-autonomous action of Sry and the non-autonomous mechanisms involving autocrine/paracrine signaling of fibroblast growth factor 9 (Fgfg) and the hormone prostaglandin $\mathrm{D} 2\left(\mathrm{PGD}_{2}\right)$ (Figure 1). In $\mathrm{XY}$ gonads, Sry is transiently activated (from E10.5 to E12.5 in mice) in the supporting cell lineage. The onset of Sry expression initiates the male differentiation pathway by upregulating Soxg [5], which in turn upregulates Fgf9 expression and increases $\mathrm{PGD}_{2}$ synthesis [6]. Fgf9 and $\mathrm{PGD}_{2}$ both help maintain Soxy expression, forming a positive feedback loop, and thereby helping to direct the differentiation of the supporting lineage into Sertoli cells. In addition, Sertoli cells are also recruited through a nonautonomous mechanism: Sry-expressing Sertoli cells secrete $\mathrm{PGD}_{2}$, which acts in a paracrine manner to upregulate Soxy expression in supporting cell precursors. This commits additional Sertoli cells to reinforce the testis fate. In parallel, Sox9 and Fgf9 downregulate female signals, such as the secreted factor Wnt4, to block ovarian differentiation.

\section{Early ovarian development}

In the absence of Sry, XX gonads develop as ovaries. Largescale transcriptional analyses have revealed that, as in $\mathrm{XY}$ gonads, a robust female-specific genetic program is initiated in XX gonads as early as E11.5 (for a review see [7]). However, no morphological differentiation is apparent until E13.5, when germ cells enter meiosis following induction by retinoic acid. In contrast to XY gonads, germ cells are crucial for the formation and maintenance of the ovarian structure in XX gonads. In their absence, follicles do not assemble and ovarian dysgenesis ensues. An 


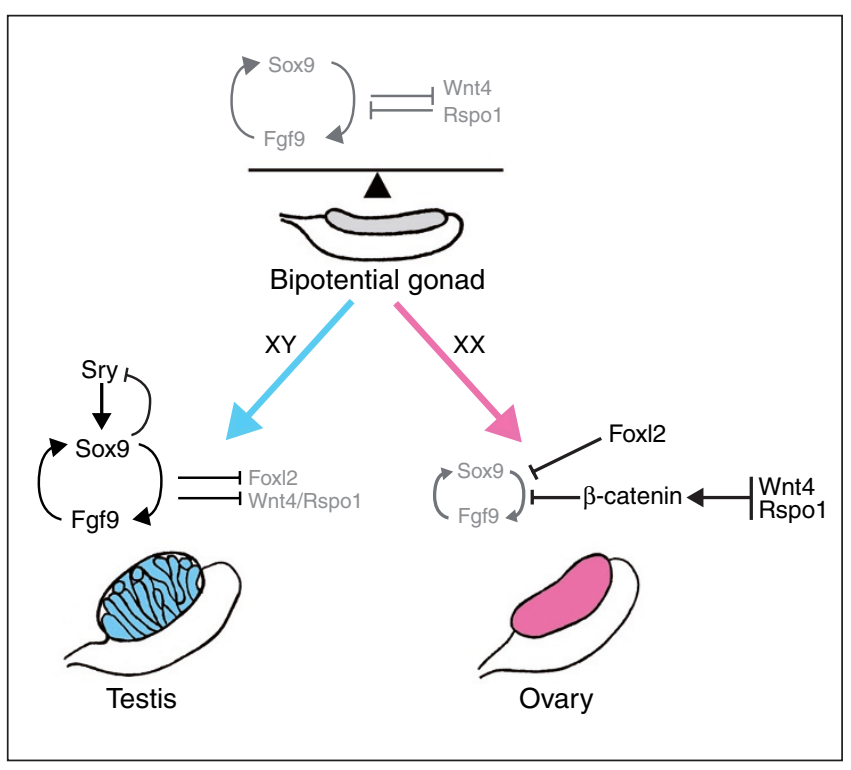

Figure 1

A genetic model for sex determination, controlled by a balance of antagonistic pathways. In XY gonads, Sry triggers upregulation of Sox9, leading to Sertoli cell commitment and testicular differentiation. Sertoli cell differentiation is a result of the establishment of a positive feedback loop between Sox9 and secretion of Fgf9 (and also PGD ; not shown), which act in a paracrine manner to recruit additional Sertoli cells. In XX gonads, two independent signaling pathways involving the Rspo1/Wnt4/ $\beta$-catenin pathway and Foxl2 tilt the balance towards the female side and silence Sox9 and Fgf9. Arrows indicate stimulation; T bars indicate inhibition.

additional modification that occurs in the nascent ovary is the formation of loose cordlike structures, referred to as the ovigerous cords. Within these cords, germ cells are interconnected via cytoplasmic bridges to form a syncitium. Around birth, some oocytes undergo apoptosis, and those that remain become surrounded by a monolayer of flattened pre-granulosa cells to form individual primordial follicles.

Over the past few years, significant progress has been made towards the identification of the molecular basis of female sex determination. Recent findings suggest that at least two independent factors or signaling pathways act in a complementary manner to promote the ovarian fate and repress male signaling and testicular development: the R-spondin1/Wnt4/ $\beta$-catenin pathway and the Foxl2 transcription factor (Figure 1).

\section{The R-spondin1/Wnt4/ $\beta$-catenin signaling pathway}

R-spondin1 (Rspo1) has recently emerged as a key femaledetermining factor, probably acting at the top of the ovarian development pathway. This secreted factor is a potent modulator of Wnt signaling and activates the canonical $\beta$-catenin pathway. Transcripts encoding Rspo1 are upregulated in somatic cells of XX gonads from E11.5 onwards. In humans, loss of function of the RSPO1 gene leads to XX female-to-male sex reversal [8], and duplications of the RSPO1 and WNT4 loci lead to XY male-tofemale sex reversal. Recent studies in mice suggest that Rspo1 is required for Wnt4 expression in XX gonads and that it exerts its functions via stabilization of $\beta$-catenin to promote ovarian fate and block testis development $[2,3]$. Targeted deletion of Rspo1 in mice impairs ovarian differentiation and triggers the development of ovotestes (gonads composed of both ovarian and testicular tissues) in XX gonads [2]. More precisely, it prevents the upregulation of Wnt4, resulting in testis-like vascularization and the appearance of androgen-producing cells, two characteristics also seen in Wnt4 mutant ovaries. Wnt4 is expressed at low levels in mouse bipotential gonads and becomes ovary-specific by E11.5. Both in humans and in mice, mutations in the Wnt4 gene lead to late-onset partial sex reversal with appearance of testis-type vasculature, adrenal-like cells and Wolffian duct derivatives [9].

Overall, Rspo1, Wnt4 and $\beta$-catenin are components of a single pathway that promotes ovarian development and suppresses the formation of testis cords. However, Rspo1 is not likely to be the sole female-determining gene because its ablation results in the formation of ovotestes. This suggests that other factor(s) or independent pathway(s) may also be involved.

\section{Foxl2 as a potential ovarian-determining factor}

In addition to the Rspo1/Wnt $4 / \beta$-catenin signaling pathway, the Foxl2 transcription factor has also been proposed as a candidate female-sex-determining gene. Foxl2 is a member of the large family of forkhead/winged-helix transcription factors and has been shown to be essential for granulosa cell differentiation and ovarian maintenance in mice. Its expression, which is independent of Rspo1, begins at E12.5 in pre-granulosa cells. Foxl2 has been implicated in XX sex reversal in goats, but its ablation in mice results in only partial secondary sex reversal, and in humans partial loss of function has been associated with premature ovarian failure. Transcriptome analyses by Garcia-Ortiz et al. [4] indicate that the ovarian genetic program in Foxl2 mutant mice is already deregulated by E13.5, long before the first observations of histological alterations and partial sex reversal. The differing effects of Foxl2 loss of function in goats, mice and humans have raised doubts about the role of Foxl2 as an ovariandetermining factor.

However, recent findings suggest that Foxl2 and the Rspo1/Wnt4/ $\beta$-catenin signaling pathway may act independently, but in a complementary manner, to promote ovarian development and suppress the male pathway. The 
combined loss of Wnt4 and Foxl2 leads to extensive gonadal $\mathrm{XX}$ sex reversal, with testis-like tubules that express high levels of Soxg and anti-Müllerian hormone and contain well differentiated spermatogonia [10]. The presence of testis cords and spermatogonia as early as birth indicates that sex reversal in double mutant ovaries is more dramatic and precocious than in either single knockout.

Nevertheless, the loss of both Wnt4 and Foxl2 does not lead to complete sex reversal but results in XX ovotestes. This suggests that additional factors or signaling pathways could also promote ovarian development and suppress the male pathway. One hypothesis proposes the existence of functional redundancy with other Wnt ligands, such as Wnt5a, Wnt6 and Wnt9a, that are also expressed in the developing ovary [7]. Alternatively, given that the ablation of Rspo1 has more dramatic effects on $\beta$-catenin activation than Wnt4 ablation, it is possible that the concomitant ablation of Rspo1 and Foxl2 could lead to a complete sex reversal.

Our knowledge of the factors and molecular pathways controlling ovarian and testicular development is steadily expanding. However, the genetic causes leading to sex determination pathologies in humans are mostly unknown. Additional genes or regulatory regions involved in the sex determination process therefore remain to be identified. Recently, large-scale genomic analyses revealed that more than $90 \%$ of the eukaryotic genome is transcribed, yielding tens of thousands of non-coding RNAs of various length. Although the function of some non-coding RNAs is understood, the biological significance of the vast majority remains unknown. Therefore, it will be particularly interesting to investigate whether these transcripts have a role in testis- and ovary-determining pathways.

\section{Acknowledgements}

We thank Dagmar Wilhelm and Marilena Papaioannou for critical comments on the manuscript. Due to journal policy, we have only sparingly referenced the literature and apologize to those whose work we were unable to specifically mention.

\section{References}

1. Fleming $A$, Vilain $E$ : The endless quest for sex determination genes. Clin Genet 2005, 67:15-25.

2. Chassot $A A$, Ranc $F$, Gregoire EP, Roepers-Gajadien HL, Taketo MM, Camerino G, de Rooij DG, Schedl A, Chaboissier MC: Activation of beta-catenin signaling by Rspo1 controls differentiation of the mammalian ovary. Hum Mol Genet 2008, 17:1264-1277.

3. Maatouk DM, DiNapoli L, Alvers A, Parker KL, Taketo MM, Capel B: Stabilization of beta-catenin in XY gonads causes male-to-female sex-reversal. Hum Mol Genet 2008, 17:29492955.

4. Garcia-Ortiz JE, Pelosi E, Omari S, Nedorezov T, Piao Y, Karmazin J, Uda M, Cao A, Cole SW, Forabosco A, Schlessinger D, Ottolenghi C: Foxl2 functions in sex determination and histogenesis throughout mouse ovary development. BMC Dev Biol 2009, 9:36.

5. Sekido R, Lovell-Badge R: Sex determination involves synergistic action of SRY and SF1 on a specific Sox9 enhancer. Nature 2008, 453:930-934.

6. Moniot B, Declosmenil F, Barrionuevo F, Scherer G, Aritake K, Malki S, Marzi L, Cohen-Solal A, Georg I, Klattig J, Englert C, Kim Y, Capel B, Eguchi N, Urade Y, Boizet-Bonhoure B, Poulat F: The PGD2 pathway, independently of FGF9, amplifies SOX9 activity in Sertoli cells during male sexual differentiation. Development 2009, 136:1813-1821.

7. Cederroth CR, Pitetti JL, Papaioannou MD, Nef S: Genetic programs that regulate testicular and ovarian development. Mol Cell Endocrinol 2007, 265-266:3-9.

8. Parma P, Radi O, Vidal V, Chaboissier MC, Dellambra E, Valentini S, Guerra L, Schedl A, Camerino G: R-spondin1 is essential in sex determination, skin differentiation and malignancy. Nat Genet 2006, 38:1304-1309.

9. Vainio S, Heikkila M, Kispert A, Chin N, McMahon AP: Female development in mammals is regulated by Wnt-4 signalling. Nature 1999, 397:405-409.

10. Ottolenghi C, Pelosi E, Tran J, Colombino M, Douglass E, Nedorezov T, Cao A, Forabosco A, Schlessinger D: Loss of Wnt4 and Foxl2 leads to female-to-male sex reversal extending to germ cells. Hum Mol Genet 2007, 16:27952804.

Published: 2 September 2009

doi:10.1186/jbiol173

(C) 2009 BioMed Central Ltd 